NASA/TM-

1998 208270

\title{
Solar Prominence Eruption
}

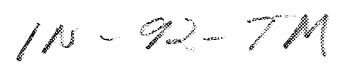

\section{Ron Moore}

An invited article for the Encyclopedia of Astronomy and Astrophysics to be published by Institute of Physics and Macmillan Publishing 


\section{Solar Prominence Eruption}

\section{Introduction}

The prominence that erupts in a prominence eruption is a magnetic structure in the chromosphere and corona. It is visible in chromospheric images by virtue of chromospheric-temperature plasma suspended in the magnetic field, and belongs to that large class of magnetic structures appropriately called filaments because of their characteristic sinewy sigmoidal form. Hence, the term "filament eruption" is used interchangeably with

the term "prominence eruption." The magnetic field holding a filament is prone to undergo explosive changes in configuration. In these upheavals, because the filament material is compelled by its high conductivity to ride with the magnetic field that threads it, this material is a visible tracer of the field motion. The part of the magnetic explosion displayed by the entrained filament material is the phenomenon known as a filament eruption, the topic of this article. Figure 1 shows a filament eruption in progress, exploding up into the corona.

This article begins with a description of basic observed characteristics of filament eruptions, with attention to the magnetic fields, flares, and coronal mass ejections in which erupting filaments are embedded. The present understanding of these characteristics in terms of the form and action of the magnetic field is then laid out by means of a rudimentary three-dimensional model of the field. The article ends with basic questions that this picture leaves unresolved and with remarks on the observations needed to probe these questions.

\section{Observed Characteristics}

Every stationary filament, whether or not it eventually erupts, stands over and tracks a neutral line (i.e., a polarity dividing line) in the photospheric magnetic flux. The width of the opposite polarity flux areas bracketing the neutral line is typically considerably greater than the height of the filament, and coronal images 
show a magnetic arcade rooted in the opposite polarities and arching high over the filament. That is, the filament is centered low in the tunnel of the coronal arch of the overall bipole, suspended in the core field, the field that is rooted near the neutral line and closely envelops the neutral line. The filament and substructure striations within the filament run nearly parallel to the neutral line. This shows that the core field threading the filament runs nearly parallel to the neutral line rather than arching right over the neutral line as it would if it were near its relaxed, minimum-energy potential configuration. In contrast to the core field, the loops of the overall arch of the bipole are more nearly orthogonal to the neutral line. Thus, the form of the filament and its location within the bipole show that the core field is greatly deformed from the potential configuration, being sheared so that it stretches along the neutral line, and that the core field is more strongly sheared than is the field farther out in the envelope of the bipole.

In a filament eruption, the active agent is the core field in and around the filament; it is the core field that explodes, exploding both itself and the filament that it carries. Thus, the size of a filament eruption is set by the length of the sheared core field traced by the filament at eruption onset. This length is a measure of the overall size of the closed magnetic bipole in which the filament and sheared core field are seated. Accordingly, the range of sizes of filament eruptions is more or less the range of the lengths of filaments found on the Sun, and this is roughly the range in the size of magnetic bipoles that occur on the Sun. This range extends from as large as half a solar radius $(\sim 300,000 \mathrm{~km})$ to as small or smaller than the larger bipoles found in the magnetic network $(<\sim 10,000 \mathrm{~km})$. The magnetic bipoles forming active regions with sunspots, and the filament eruptions in these regions, are usually medium sized, typically $\sim 30,000 \mathrm{~km}$, but ranging up to as large as $100,000 \mathrm{~km}$ at eruption onset. The filament eruption in Figure 1 is seated in an active region and is larger than average: the erupting filament and sheared core field were initially $\sim 50,000 \mathrm{~km}$ long. Although the largest (in span) of all filament eruptions do not occur in active regions, the larger of the filament eruptions in active regions, in particular filament eruptions from between large impacted opposite-polarity sunspots, mark the Sun's most powerful magnetic explosions. This is so because sunspots have the strongest magnetic fields found on the Sun, and consequently the sheared core fields between the largest impacted sunspots have the largest store of nonpotential magnetic energy. The very largest filaments and filament eruptions are in quiet regions, 
on the neutral lines of the bipolar arcades under large coronal streamers. The strength of the filament core field in these is only $\sim 10 \mathrm{G}$, a hundred times less than in the most powerful filament eruptions from large sunspot regions. The eruption of one of these largest quiet-region filaments is spectacular, as in the example shown in Figure 2, even though the total energy of the explosion is 10 to 100 times less than for the strongest ones in active regions. While the largest filament eruptions occur in only the largest bipoles, which are all in quiet regions, small filament eruptions can occur wherever small bipoles occur, which can be within active regions as well as in quiet regions. However, most of the smallest filament eruptions (from bipoles $<\sim 10,000 \mathrm{~km}$ across) occur in quiet regions, due to the ubiquity of such bipoles in the magnetic network. It is likely that many macrospicules are small $(\sim 10,000 \mathrm{~km})$ filament eruptions, and this raises the possibility that many spicules (which are rooted in the network and are similar to macrospicules but smaller) are still smaller filament eruptions.

There is enough magnetic flux all over the Sun, in all quiet regions as well as in active regions, that the entire solar atmosphere above the photosphere is permeated with magnetic field strong enough to impose its structure on the chromosphere and corona. Consequently, none of the bipoles in which filaments and filament eruptions are seated is totally isolated, but is hemmed in to some degree by adjacent magnetic fields. When these adjacent fields are weak relative to the eruption bipole, the eruption proceeds about as it would if there were no adjacent field. Often, some of the adjacent fields, weak or strong, are in other bipoles that have their own sheared core fields. In these cases, a filament eruption in one bipole can trigger and interact with another eruption in one or more adjacent bipoles. The morphology and development of these multiple-bipole eruptions is naturally more complex than in single-bipole events. All of the figures in this article show examples or models of single-bipole events.

The filament eruption in any one bipole, whether or not it is part of a multiple-bipole eruption, is either ejective or confined. In an ejective eruption, the erupting core field becomes an expanding, twisted, arched flux rope, the top of which escapes far out of the originally closed bipole while the ends remain rooted in the surface. This requires that the envelope of the bipole also eject along with the filament flux rope, the filament 
rope riding inside the distending bubble of envelope field. The filament eruptions in Figures 1 and 2 are ejective. In a confined eruption, there is no distinct formation of a monolithic rising flux rope. Instead, there is complex eruptive untwisting and restructuring of the core field as the erupting core field undergoes an overall expansion. The whole eruption is confined and arrested within the enveloping bipole, and the overall magnetic arch of the bipole remains closed throughout the eruption. Figure 3 shows a confined filament eruption from beginning to end. This eruption spans the interior of an active region; so it is of average size and is seated in strong field. As do ejective eruptions, confined filament eruption occur in quiet regions as well as in active regions, over the same wide range of size and field strength. The factors that dictate whether an eruption is to be confined or ejective are probably specifics of the pre-eruption field configuration and the strength of the envelope field relative to the core field.

Every filament eruption, confined or ejective, is accompanied by increased coronal heating in the bipole in which it is seated, and every ejective filament eruption, in addition, accompanies the ejection of the corona in and above the bipole, i.e., is an intrinsic part of a larger surrounding coronal mass ejection, the core of which is the ejected filament. Figure 4 shows the coronal mass ejection bubble around an ejected quiet-region filament. The bright twisted loop in the core of the bubble is the filament flux rope from a quiet-region filament eruption like that in Figure 2, but at a later phase, much farther out in the corona. The corresponding coronal mass ejections for ejective filament eruptions from active regions are similar in overall form to those from quiet regions, but usually accelerate more rapidly to final speeds that are $2-5$ times faster (as fast as $1000-2000 \mathrm{~km}$ $\mathbf{s}^{\wedge}-1$ ). Because these fast ejective filament eruptions are always accompanied by a strong flare in the ejective bipole, they are aptly called flare sprays. The burst of heating in any large strong-field filament eruption in an active region produces a major flare in coronal emission, increasing the Sun's total X-ray luminosity by a factor of 10 to 1000 . In these eruptions and in many quiet-region eruptions, the enhanced coronal heating begins in the erupting core field at eruption onset, rendering this field visible in coronal images. Coronal images during the onset phase often show the whole core field to have the overall shape of an S or backward S. Later in ejective eruptions, after the filament flux rope has been ejected far out of the bipole, the coronal images show a bright, growing coronal arcade straddling the neutral line. In large weak-field ejective eruptions like those in 
Figures 2 and 4, the coronal heating can be so weak as to be hardly noticeable in the global X-ray luminosity, but is usually strong enough to be seen in coronal X-ray images, at least in the post-ejection coronal arcade. In active-region eruptions the heating also produces flare brightening of the chromosphere at the feet of the field lines threading the coronal heating. In confined eruptions and early in ejective eruptions, this chromospheric brightening occurs close along the neutral line under the filament, as the filament erupts (as in Figures 1 and 3). In confined eruptions, coronal images show the filament core field brightening and expanding as it transforms into an arch standing on the chromospheric brightening, as does the chromospheric arch that appears in Figure 3 as the eruption ends. In ejective eruptions after the filament is ejected, the chromospheric flare brightening is in two separating ribbons at the feet of the coronal arcade.

\section{Form, Energy, and Action of the Magnetic Field}

The filament eruptions in flares and coronal mass ejections, together with magnetograms (maps of the strength and distribution of the photospheric roots of the magnetic field) reveal that these events are fundamentally magnetic explosions, and that the exploding magnetic field has the following characteristics: (1) the pre-eruption field is a closed bipole; (2) the field along the neutral line through the core of the bipole is greatly sheared and has an overall sigmoidal form, so that it traces the neutral line through the bipole and has oppositely curved elbows on opposite ends; (3) the pre-eruption core field has a large store of nonpotential magnetic energy; (4) much of this energy is released by the untwisting and expansion of the filament core field as it erupts; and (5) the released energy is comparable to the total energy expended in the accompanying flare and/or coronal mass ejection (up to $\sim 10^{\wedge} 32 \mathrm{erg}$ in the largest active-region events). The evidence for the field configuration and its transformation in the filament-eruption explosion is outlined in the previous section. The sketches in Figure 5 show the three-dimensional form, action and reconnection of the magnetic field implied by the observed characteristics of filament eruptions and their observed involvement in flares and coronal mass ejections. In confined eruptions, the overall bipole remains closed, containing and arresting the eruption within

it. In ejective eruptions, the entire bipole eruptively opens and simultaneously closes via the sequence and 
topology of the reconnection. The expanding ejected filament flux rope and envelope field can continue on to become a coronal mass ejection that blasts out into the solar wind.

\section{Current Research}

The model sketched in Figure 5 displays, for single-bipole filament eruptions, the typical three-dimensional form of the magnetic field and its change in topology as it explodes, to the extent that these have been revealed by the best magnetograms and chromospheric images from the ground together with the best transition-region and coronal images from space. These observations indicate that reconnection occurs, beginning low in the sheared core field early in the eruption, but it is not clear why the reconnection starts or whether the reconnection is essential for the explosion. Basic questions left unresolved by present observations are:

1. How does the metastable pre-eruption field configuration originate?

2. How does the configuration destabilize to explode?

3. Is reconnection necessary for either the triggering or the growth of the explosion?

The most needed observations for answering these questions are vector magnetograms of substantially better sensitivity and spatial resolution than are now possible from the ground. Vector magnetograms provide a direct measure of the shear in the magnetic field in the photosphere. To probe why and how filaments erupt, the vector magnetograms need to track the development of the entire bipole in which the sheared core field builds up and explodes. It is anticipated that the needed improved vector magnetograms, along with simultaneous high-resolution images of the chromosphere, transition region, and corona, will be provided from space by the Solar-B Mission presently being developed by Japan, jointly with the United States and the United Kingdom, for launch 2004. 
Bibliography

Kahler S W 1992 Solar flares and coronal mass ejections Ann. Rev. Astron. Astrophys. 30 113-141

Moore R L 1988 Evidence that magnetic energy shedding in solar filament eruptions is the drive in accompanying flares and coronal mass ejections Astrophys. J. 324 1132-1137

Moore R L, Schmieder B, Hathaway D H and Tarbell T D 1997 3-D magnetic field configuration late in a large two-ribbon flare Solar Phys. 176 153-169

Rust D M, Hildner E, Dryer M, Hansen R T, McClymont A N, McKenna Lawlor S M P, McLean D J, Schmahl E J, Steinolfson R S, Tandberg-Hanssen E, Tousey R, Webb D F and Wu S T 1980 Mass ejections In:

Sturrock, P A (ed.) Solar Flares (Boulder, Colorado: Colorado Associated University Press) pp 273-339

Svestka Z, Jackson B V and Machado M E 1992 Eruptive Solar Flares (Berlin: Springer-Verlag)

\author{
Ron Moore \\ NASA/Marshall Space Flight Center \\ Solar Physics Branch ES82 \\ Huntsville, AL 35812, USAPeafc
}




\section{Figure Captions}

Figure 1. An ejective filament eruption exploding out of an active region near the limb. The expanding filament magnetic flux rope is seen to have overall twist and complex substructure. At this point, about 10 minutes into the explosion, the filament flux rope has increased in volume by a factor of $\sim 1000$. This photograph is an H-alpha filtergram (from Big Bear Solar Observatory); it shows plasma in the erupting filament that is at chromospheric temperatures $(5,000-10,000 \mathrm{~K})$.

Figure 2. A large filament ejectively erupting from a quiet region on the limb. The many long striations show that the filament material is strung in a twisted magnetic flux rope. This image is a He II $304 \mathrm{~A}$ spectroheliogram (from the Naval Research Laboratory's extreme ultraviolet spectroheliograph on Skylab); it shows plasma at transition-region temperatures $(\sim 80,000 \mathrm{~K})$.

Figure 3. A confined filament eruption in an active region. In this 20 minute sequence, the filament core field untwists and expands as it erupts to become a magnetic arch in the interior of this overall bipolar active region. The active-region's opposite-polarity sunspots are seen at opposite ends of the erupting filament and final arch. These images are blue-wing H-alpha filtergrams from Big Bear Solar Observatory.

Figure 4. The filament flux rope in the core of the coronal mass ejection from a large quiet-region ejective filament eruption. The ejecting magnetic field, which is traced by and carries the ejecting plasma within it, is seen in the act of exploding out through the corona and into the solar wind. The occulting disk extends half a solar radius above the limb. This white-light image is from the High Altitude Observatory's coronagraph on the Solar Maximum Mission.

Figure 5. Three-dimensional sketches of the magnetic field before and during filament eruptions. The dashed curve is the polarity neutral line in the photosphere. The diagonally-lined object in the upper left panel is the pre-eruption filament; for clarity of the eruption of the core field that carries the erupting filament material, this 
material is not shown in the other panels. The gray areas are chromospheric flare brightenings. The ragged arc in the background in the chromospheric limb. Early in the eruption, reconnection occurs in the sheared core field in or under the filament, as in the upper right panel. In ejective eruptions, after the filament flux rope forms and ejects, the ejected envelope field of the pre-eruption arcade is wrapped around the filament core flux rope and forms much of the surrounding expanding coronal mass ejection bubble that envelops the filament core rope, and the stretched legs of the envelope field reconnect to form the growing coronal arcade rooted in the separating chromospheric flare ribbons, as in the lower right panel. 


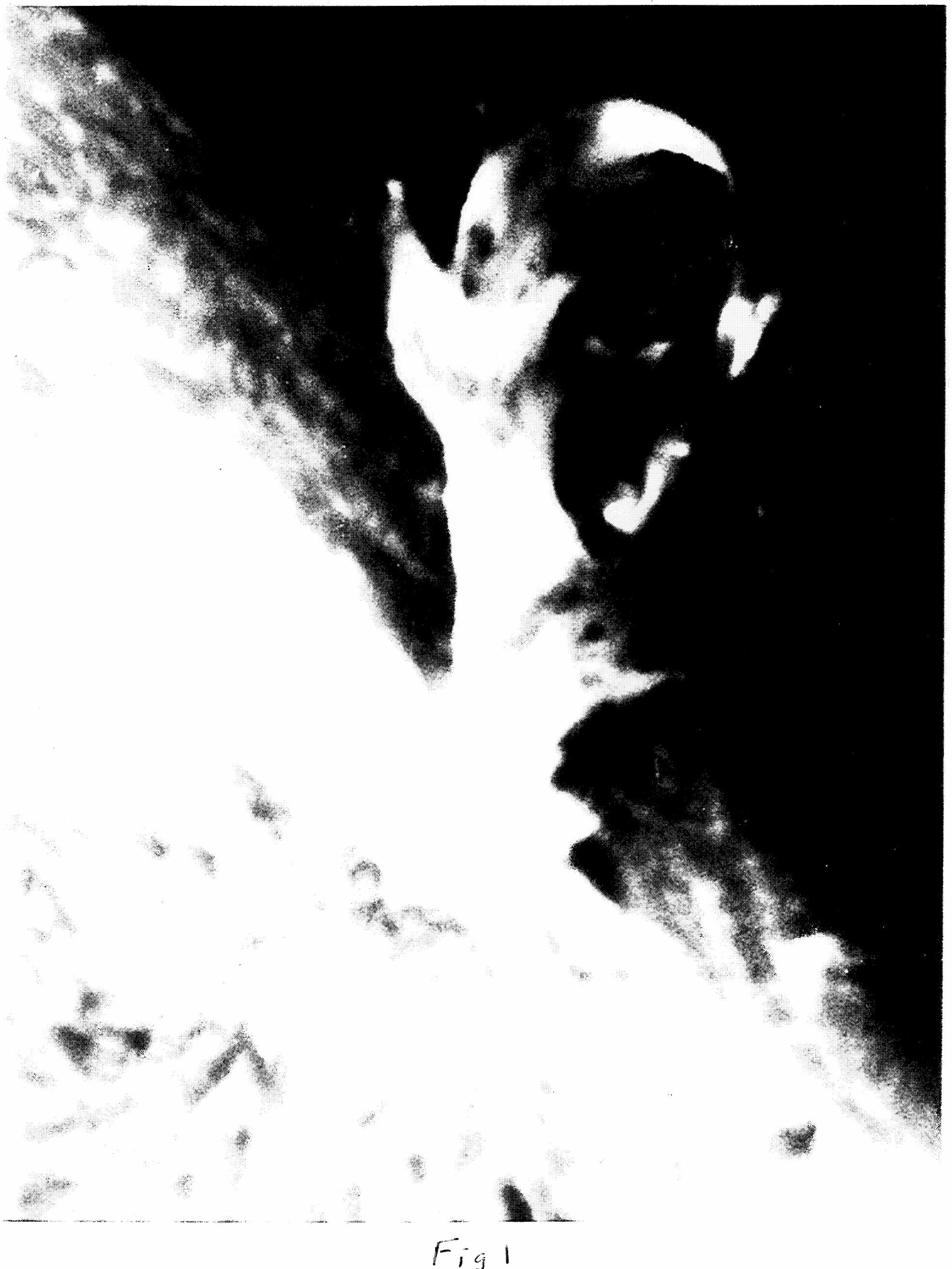




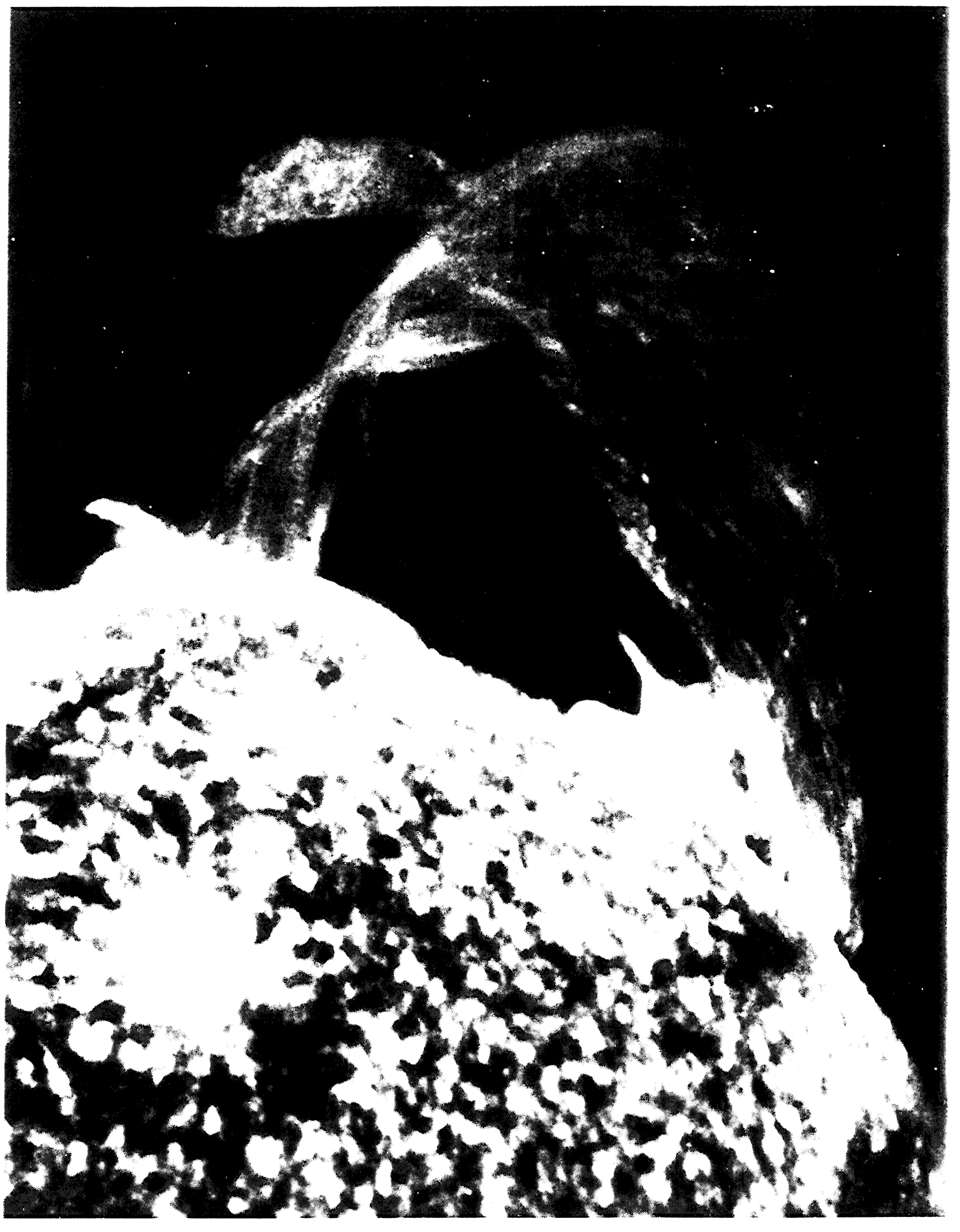



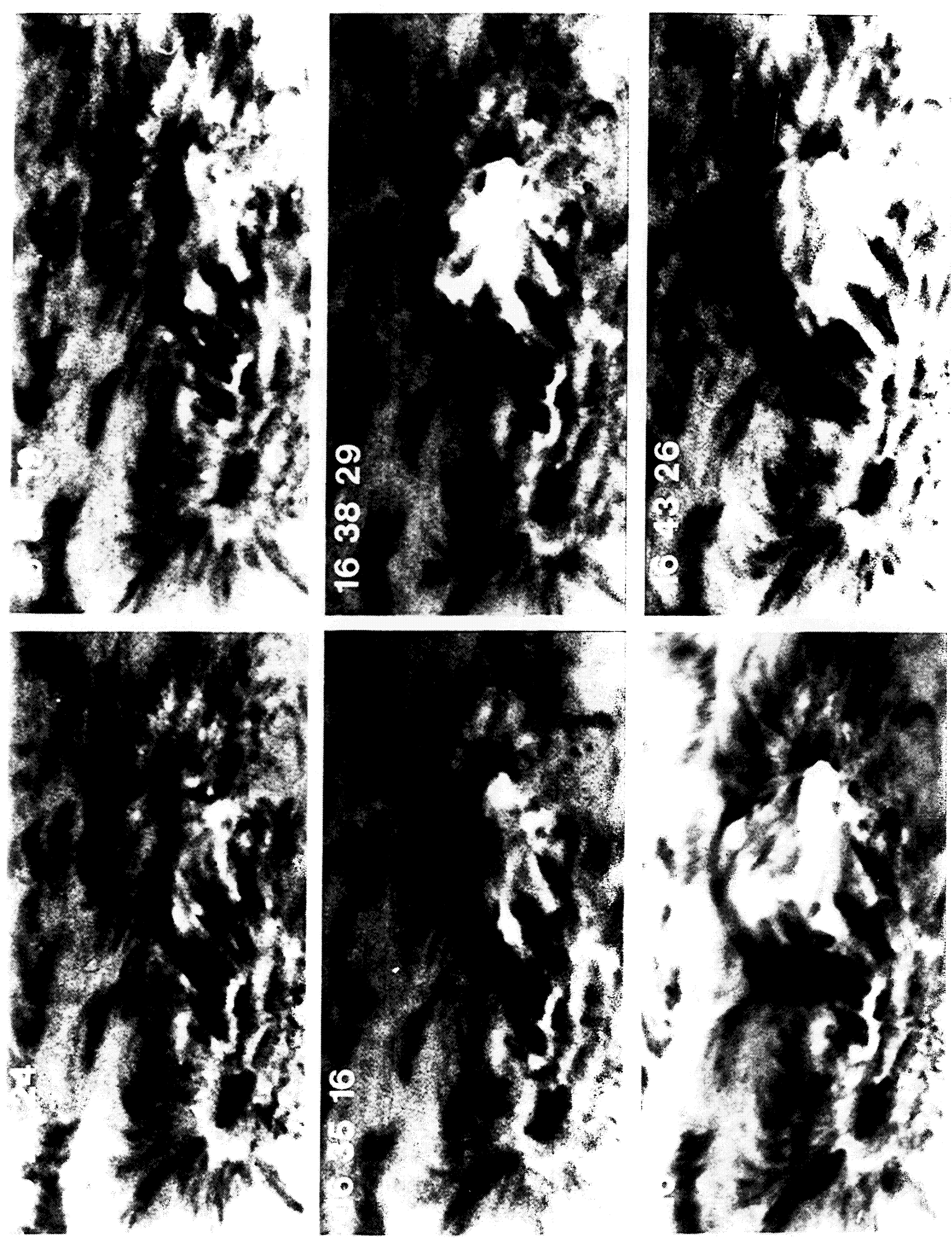


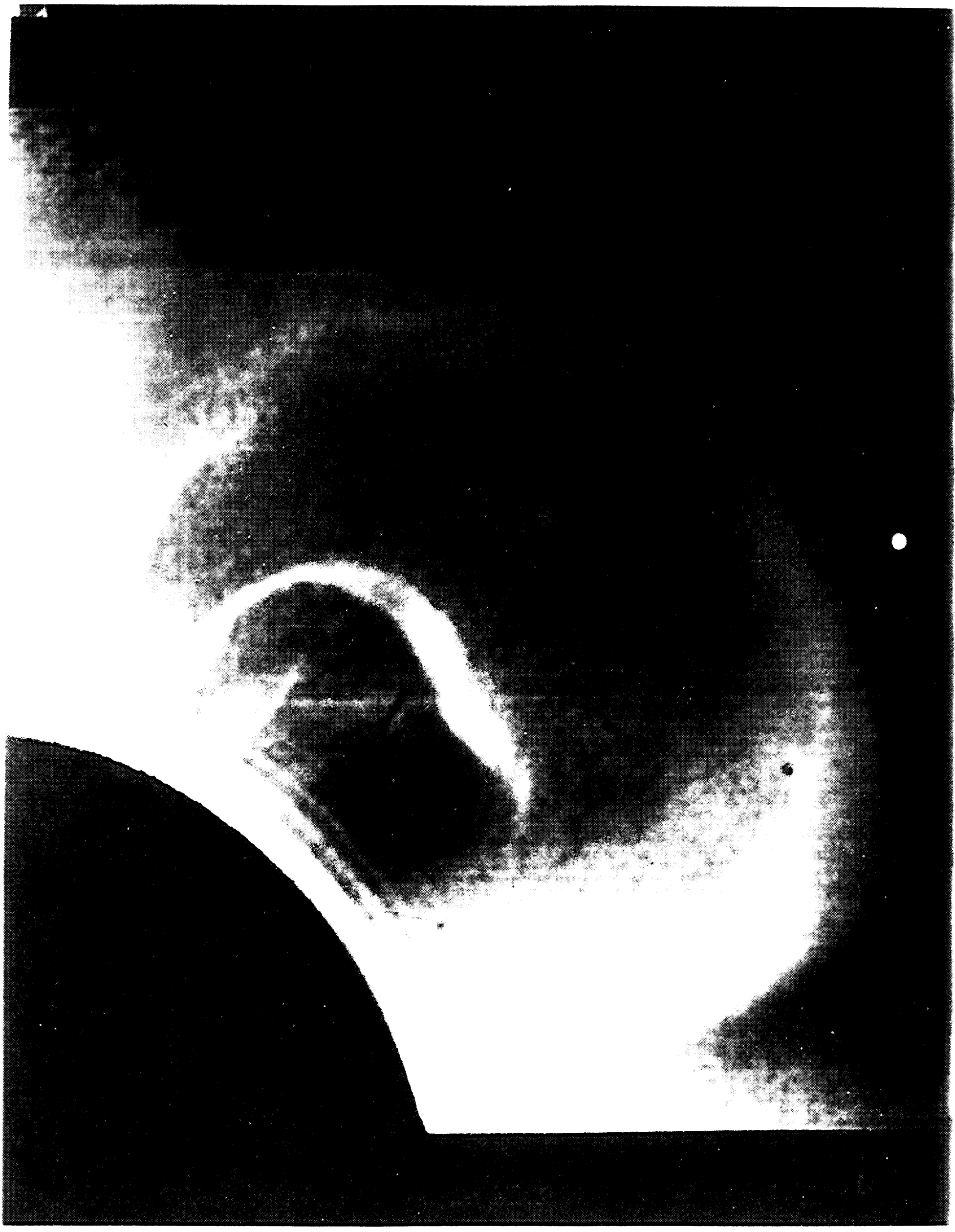

$1-94$ 


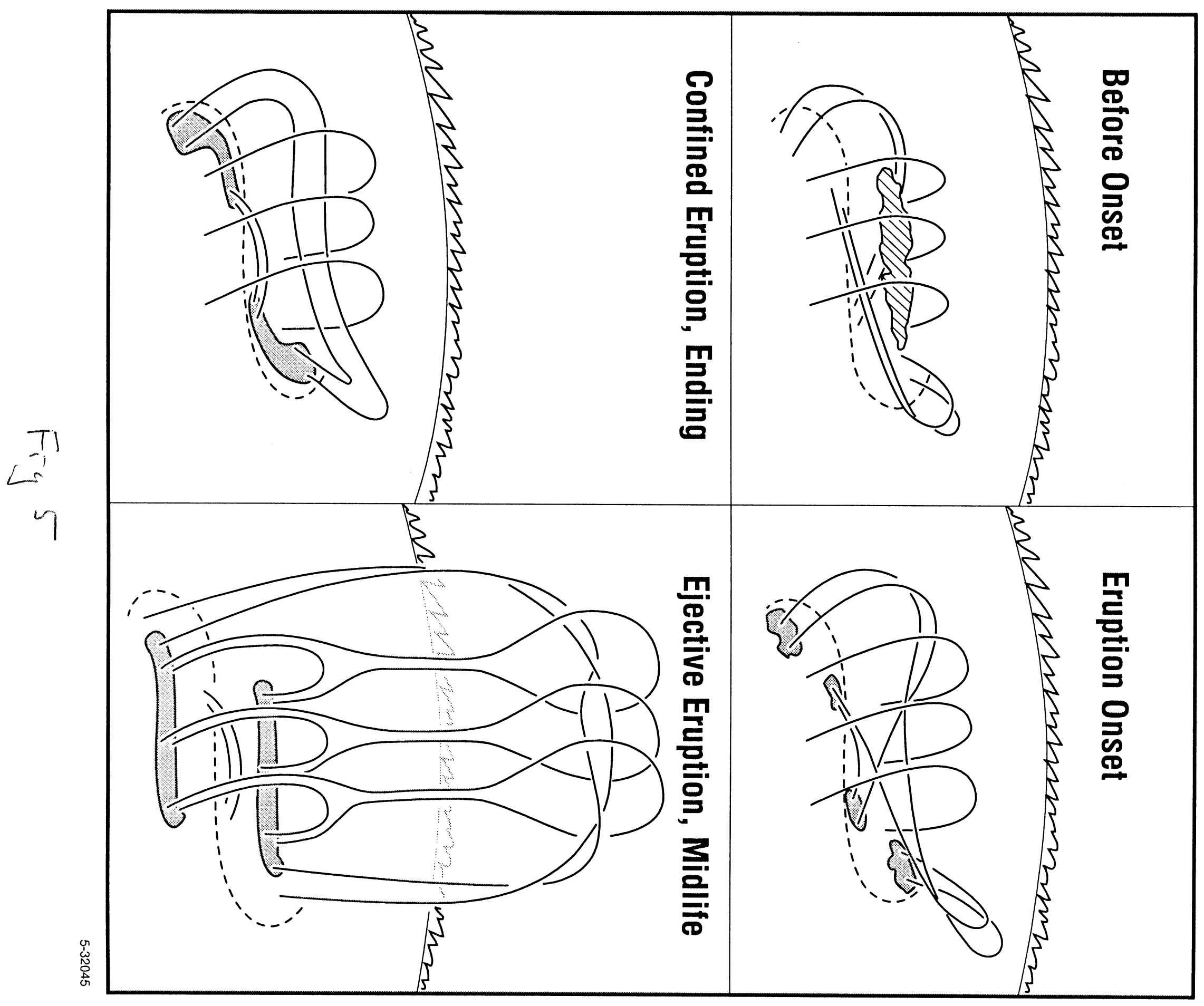




\section{Abstract}

\section{Solar Prominence Eruption}

The prominence that erupts in a prominence eruption is a magnetic structure in the chromosphere and corona. It is visible in chromospheric images by virtue of chromospheric-temperature plasma suspended in the magnetic field, and belongs to that large class of magnetic structures appropriately called filaments because of their characteristic sinewy sigmoidal form. Hence, the term "filament eruption" is used interchangeably with the term "prominence eruption". The magnetic field holding a filament is prone to undergo explosive changes in configuration. In these upheavals, because the filament material is compelled by its high conductivity to ride with the magnetic field that threads it, this material is a visible tracer of the field motion. The part of the magnetic explosion displayed by the entrained filament material is the phenomenon known as a filament eruption, the topic of this article. Figure 1 shown a filament eruption in progress, exploding up into the corona.

This article begins with a description of basic observed characteristics of filament eruptions, with attention to the magnetic fields, flares, and coronal mass ejections in which erupting filaments are embedded. The present understanding of these characteristics in terms of the form and action of the magnetic field is then laid out by means of a rudimentary three-dimensional model of the field. The article ends with basic questions that this picture leaves unresolved and with remarks on the observations needed to probe these questions. 